\title{
Surface nanoscale axial photonics structures introduced by bending of optical fibers
}

D. Bochek, N. A. Toropov, Z. Han, I. Vatnik, M. Sumetsky

D. Bochek, N. A. Toropov, Z. Han, I. Vatnik, M. Sumetsky, "Surface nanoscale axial photonics structures introduced by bending of optical fibers," Proc. SPIE 10681, Micro-Structured and Specialty Optical Fibres V, 106810F (9 May 2018); doi: 10.1117/12.2306949

SPIE. Event: SPIE Photonics Europe, 2018, Strasbourg, France 


\title{
Surface nanoscale axial photonics structures introduced by bending of optical fibers
}

\author{
D. Bochek ${ }^{\mathrm{a}}$, N. A. Toropov ${ }^{\mathrm{b}}$, Z. Han ${ }^{\mathrm{a}}$, I. Vatnik ${ }^{\mathrm{a}}$, and M. Sumetsky \\ ${ }^{a}$ Novosibirsk State University, Pirogova str. 2, Novosibirsk 630090, Russia; \\ ${ }^{\mathrm{b}}$ Aston Institute of Photonic Technologies, Aston University, Birmingham, B4 7ET, United \\ Kingdom
}

\begin{abstract}
The new manufacturing method for fabrication of Surface Nanoscale Axial Photonics (SNAP) structures has been developed. We showed experimentally that the bent fiber can achieve the nanometer-scale variation in the effective fiber radius sufficient for fabrication of SNAP microresonators. The advantage of the demonstrated method is in its simplicity, robustness, and mechanical tunability of the fabricated devices.
\end{abstract}

Keywords: Optical fiber, micro-optical devices, whispering gallery modes, microresonators, surface nanoscale axial photonics, elasto-optic effect

\section{INTRODUCTION}

Surface Nanoscale Axial Photonics (SNAP) is the ultraprecise platform for fabrication of miniature optical structures at the surface of optical fibers [1]. In SNAP structures, whispering gallery modes (WGMs) circulate around the optical fiber surface and undergo slow axial propagation. The WGM propagation is controlled by nanoscale effective radius variation (ERV) of the optical fiber, which includes the contribution from the variations of refractive index and physical dimensions of the fiber (see Eq. (5) below). The propagation is described by the one-dimensional Schrödinger equation. SNAP devices can be manufactured with unprecedented sub-angstrom precision [2] by local annealing with focused $\mathrm{CO} 2$ laser radiation [1] and by the femtosecond laser inscription [3]. The subangstrom fabrication precision of SNAP devices and their ultralow loss allows one to create complex miniature photonic circuits having promising applications in communication technologies, quantum computing, optomechanics, microfluidics, and sensing.

In the present work we suggest the new manufacturing method for fabrication of SNAP structures. Our approach is much simpler than those developed previously and consists in controllable fiber bending. We showed numerically and experimentally that the bent fiber can achieve the required nanometer-scale variation in the effective fiber radius. This ERV appears due to elasto-optic effect.

Remarkably, due to the positive sign of the ERV, this local bending introduces a SNAP optical bottle resonator [1] which parameters can be tuned by variation of the bending radius. Generally, more complex ERV can be introduced by advanced manipulation of the fiber ends. In addition, the suggested approach can be applied for tuning of SNAP structures which are fabricated by the methods developed previously.

\section{SIMULATIONS IN COMSOL}

To create a SNAP structure, a slow nanoscale variation of the effective radius variation is needed. We used COMSOL simulations to determine required bending radius for standard SMF-28 optical fiber with refractive index $n_{0}=1.45$ and dependence of ERV on curvature. Since WGMs circulate around the fiber surface, we calculated the stress tensor integrals along its path represented by the circumference, as shown in Fig. 1 (b). At each point of the WGM path, the three principal stresses $\sigma_{x}, \sigma_{y}$ and $\sigma_{z}$ were found, which are related to the principal indices of refraction $n_{x}, n_{y}$ and $n_{z}$ through known elasto-optical coefficients $C_{1}$ and $C_{2}$ (see, e.g., [4]): 


$$
\begin{gathered}
\left\{\begin{array}{l}
n_{x}=n_{0}+C_{1} \sigma_{x}+C_{2}\left(\sigma_{y}+\sigma_{z}\right) \\
n_{y}=n_{0}+C_{1} \sigma_{y}+C_{2}\left(\sigma_{x}+\sigma_{z}\right) \\
n_{z}=n_{0}+C_{1} \sigma_{z}+C_{2}\left(\sigma_{x}+\sigma_{y}\right)
\end{array}\right. \\
C_{1}=-0.65 \cdot 10^{-12}\left(\frac{1}{P a}\right), \quad C_{2}=-4.22 \cdot 10^{-12}\left(\frac{1}{P a}\right)
\end{gathered}
$$

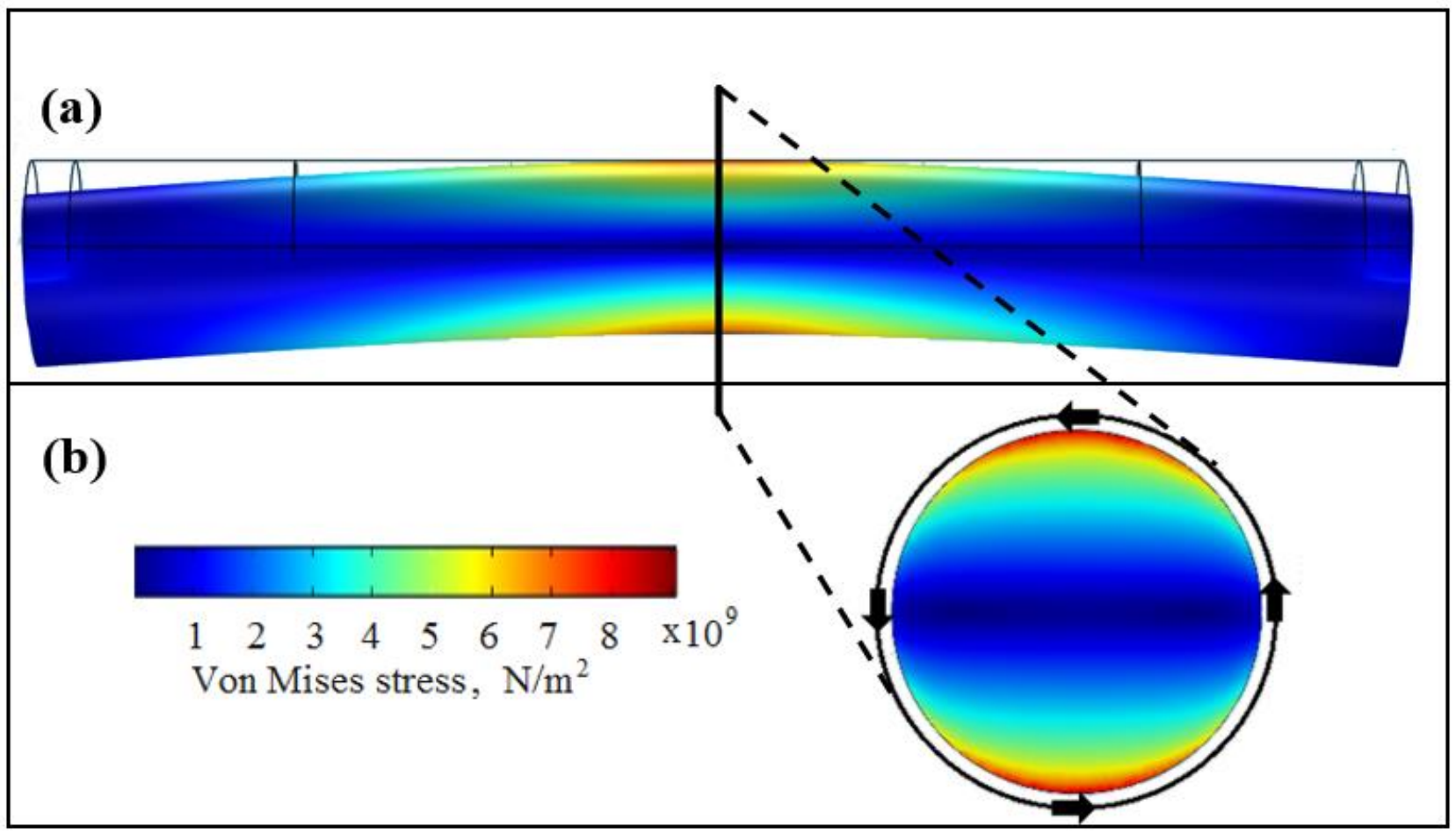

Figure 1. COMSOL simulation of bent optical fiber. Blocks (a) and (b) represents stress distribution in different projections. Arrows show the way of integration of the refractive index.

Our numerical results show that bending of the silica fiber with realistic bending radius $R$ can cause the increase of the effective fiber radius by several nanometers, which is sufficient to introduce SNAP resonators. For example, the bending of SMF-28 $125 \mu \mathrm{m}$ diameter silica fiber with curvature radius $1 \mathrm{~cm}$ introduces the positive ERV equal to $4 \mathrm{~nm}$. Simple estimates support our numerical results. The contribution of strain and stress into ERV calculated using the beam bending theory in the linear in curvature $R^{-1}$ approximation [5] vanish. However, in the quadratic approximation, the relative change of the fiber radius due to bending can be estimated by an order of magnitude as

$$
\frac{\Delta r}{r_{0}} \sim \frac{r_{0}^{2}}{R^{2}}
$$

where $r_{0}$ is the fiber radius and $R$ is the characteristic radius of curvature of the fiber. The corresponding change of the refractive index due to the stress optic effect

$$
\Delta n \sim C_{2} E \frac{\Delta r}{r_{0}},
$$

where $E$ is the Young modulus. For the silica fiber $E \approx 70 \mathrm{GPa}$ so that $C_{2} E \sim\left(4 \cdot 10^{-12}\right) \cdot\left(7 \cdot 10^{10}\right) \sim 0.3$ and the contribution of the physical change of the fiber radius $\Delta r$ and the change of its refractive index $\Delta n$ due to the stress optic effect to the ERV 


$$
\Delta r_{e f f}=r_{0}\left(\frac{\Delta r}{r_{0}}+\frac{\Delta n}{n}\right)
$$

have the same order of magnitude. For example, the SMF-28 fiber with radius $r_{0}=62.5 \mu \mathrm{m}$ bent with the characteristic curvature radius $R=3 \mathrm{~mm}$, has $\Delta r_{e f f} \approx 30 \mathrm{~nm}$ while the corresponding resonance wavelength variation [6]

$$
\Delta \lambda=\lambda_{0} \frac{\Delta r_{e f f}}{r_{0}}
$$

is $0.7 \mathrm{~nm}$ at wavelength $\lambda_{0}=1.5 \mu \mathrm{m}$.

Generally, the dependence of the ERV on the shape of the bent fiber is nonlocal and can be determined by the solution of equations from nonlinear elastic theory accounting for all terms quadratic in fiber curvature. Accurate solution of this problem will be considered elsewhere.

\section{EXPERIMENTAL RESULTS}

To measure the WGMs spectra of the bent optical fiber, it was fixed in a narrow tube, thereby acquiring the shape of a loop as it demonstrated in Fig. 2. In such configuration, the fiber curvature has a smooth distribution with a maximum at the center of the loop.

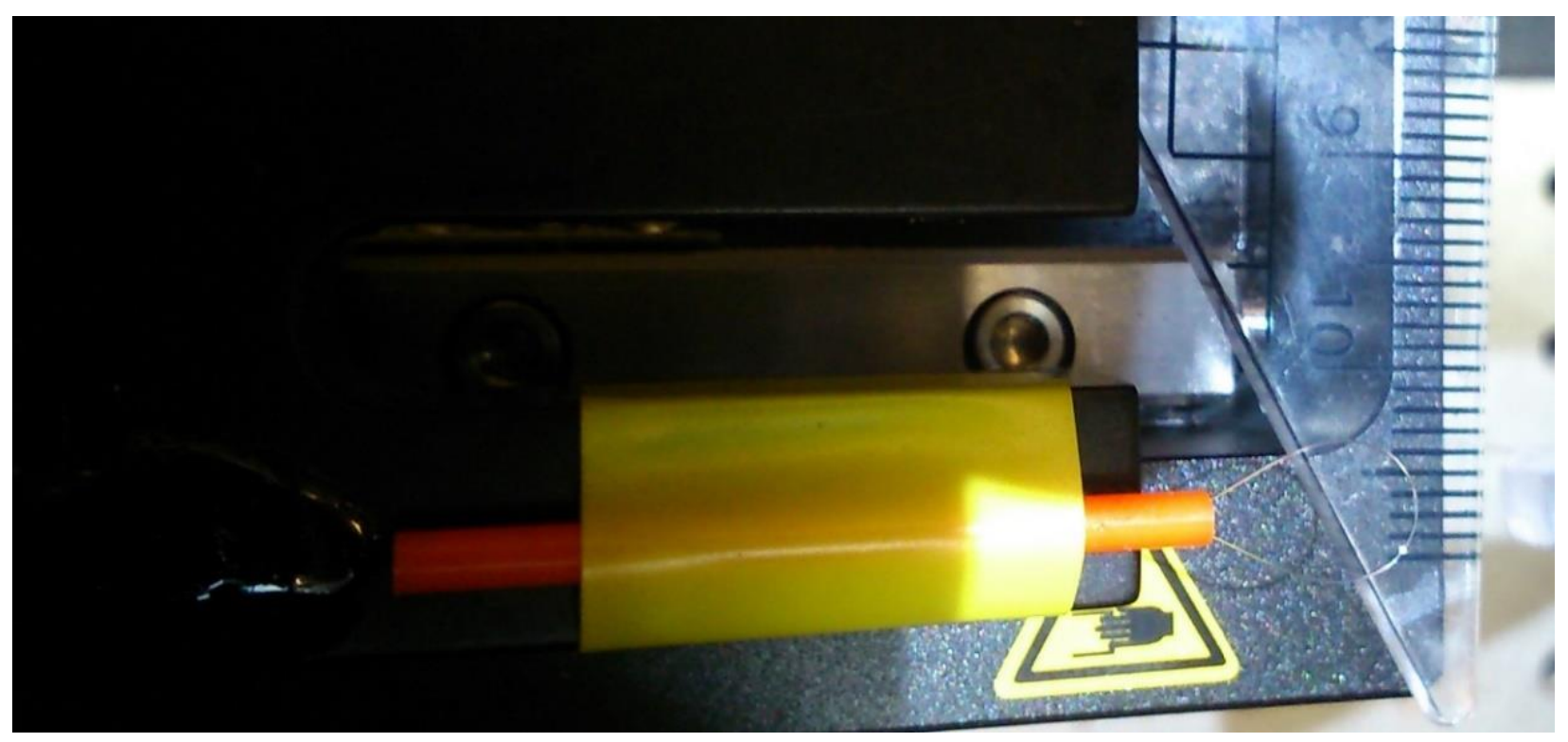

Figure 2. Photo of optical fiber loop fixed on a 3D stepper motor.

WGMs can be excited in an optical fiber using a microfiber (MF) [7], specifically, a micrometer diameter waist of a biconical fiber taper (Fig. 3.), which is attached normal to the loop and connected to the light source and detector. The spectrum was measured almost along the length of the loop in steps of $250 \mu \mathrm{m}$. The surface plot of transmission spectra of resonator introduced by bending of the fiber is shown in Fig. 4.

The introduced ERV $\Delta \mathrm{r}$ is related to the resonant wavelength variation $\Delta \lambda$ by the rescaling relation defined by Eq. (6), where the fiber radius $r_{0}=62.5 \mu \mathrm{m}$ and wavelength $\lambda_{0}=1552.1 \mathrm{~nm}$. From this relation, the maximum wavelength variation $\Delta \lambda=0.3 \mathrm{~nm}$ in Fig. 4 corresponds to the ERV $\Delta r=12.1 \mathrm{~nm}$. 


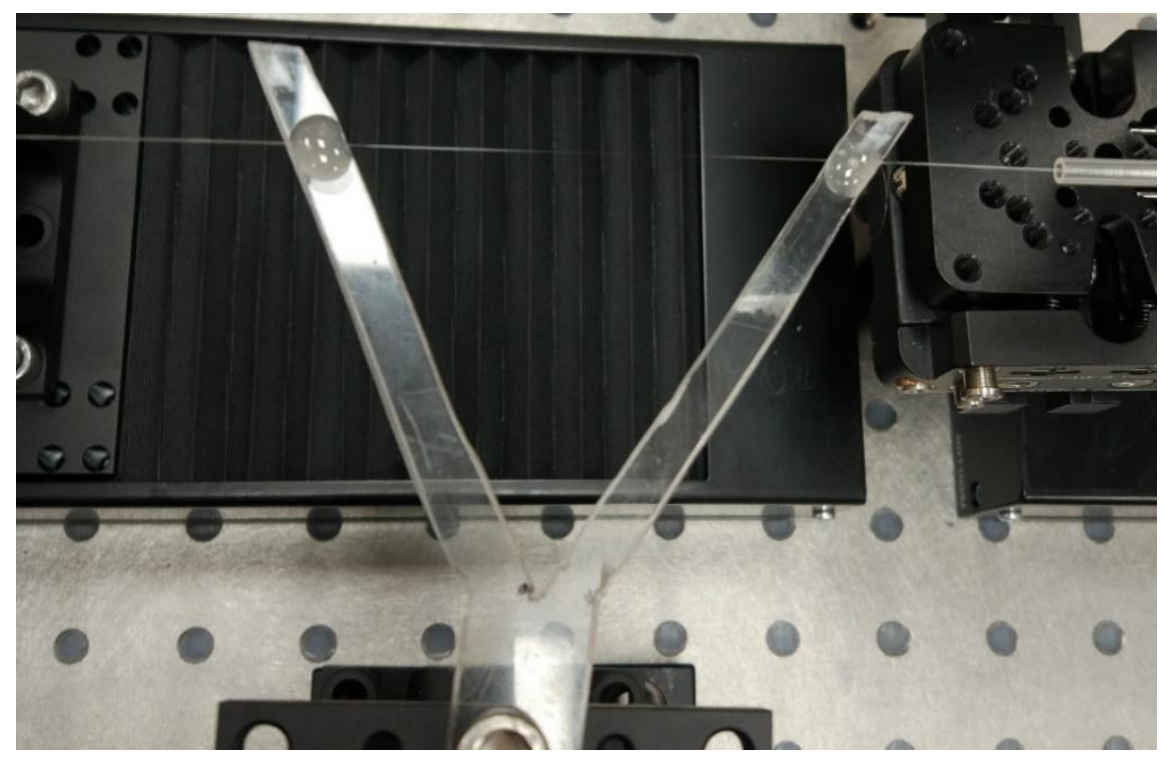

Figure 3. Photo of biconical fiber taper with $4 \mu \mathrm{m}$ diameter waist for exciting WGMs in the fiber loop.

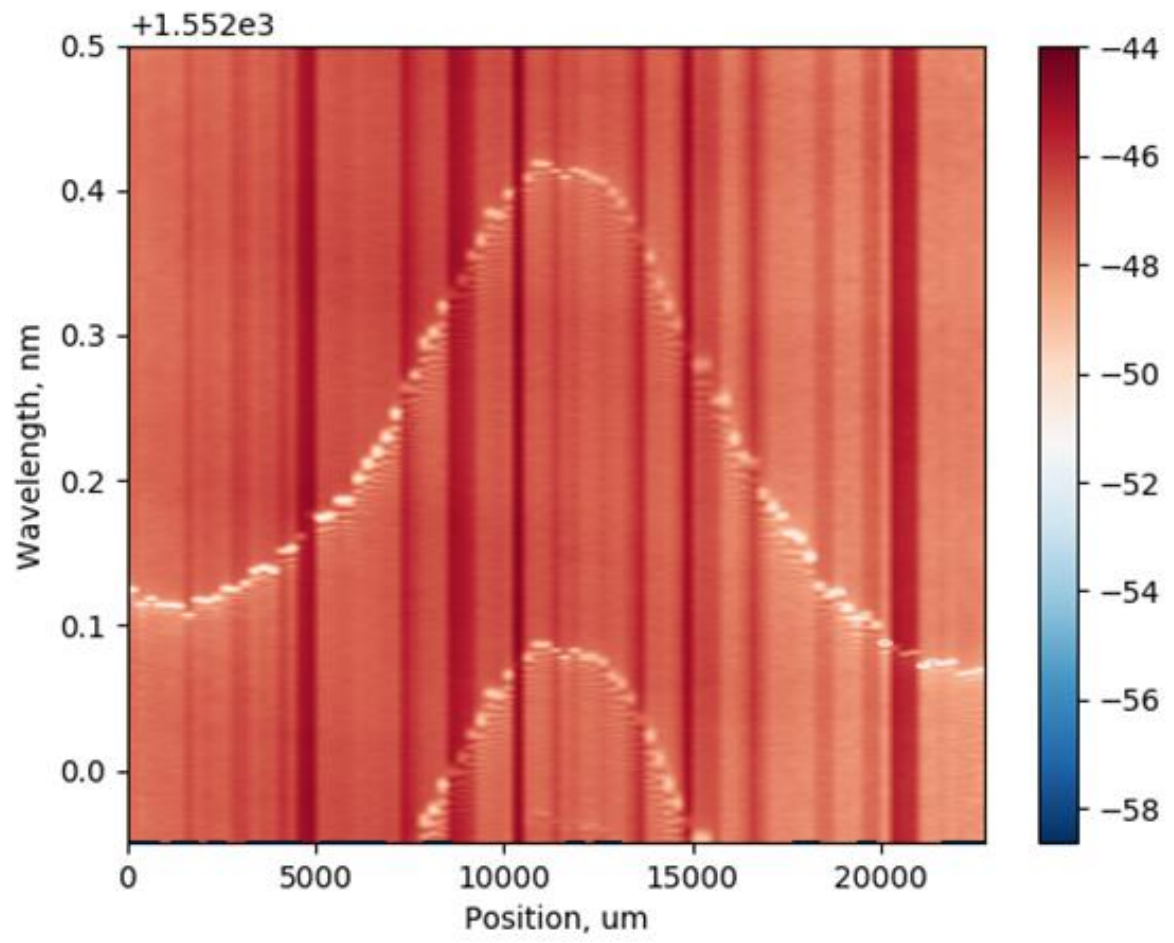

Figure 4. Surface plot of resonant transmission spectra measured with $250 \mu \mathrm{m}$ step along the fiber loop.

The coordinates of the contact between the microfiber and the loop are shown in Fig. 5(a) as red points. The shape of the loop is well approximated by a parametric curve of the following form, which is represented by blue line:

$$
\left\{\begin{array}{l}
x(t)=[(\sin (3 t) \cdot a-b) \cdot \cos (t)] \cdot c-d \\
y(t)=[(\sin (3 t) \cdot a-b) \cdot \sin (t)] \cdot f-g
\end{array},\right.
$$

where constants $a=0.48 \mathrm{~mm}, b=0.7 \mathrm{~mm}, c=9.15 \mathrm{~mm}, d=1.71 \mathrm{~mm}, f=7.8 \mathrm{~mm}$ and $g=0.73 \mathrm{~mm}$ are determined by fitting the experimental data. 
We used the Eq. 7 to determine the ERV dependence on the bending curvature. The dependence of the curvature on the optical fiber length is shown in Fig. 5(b).
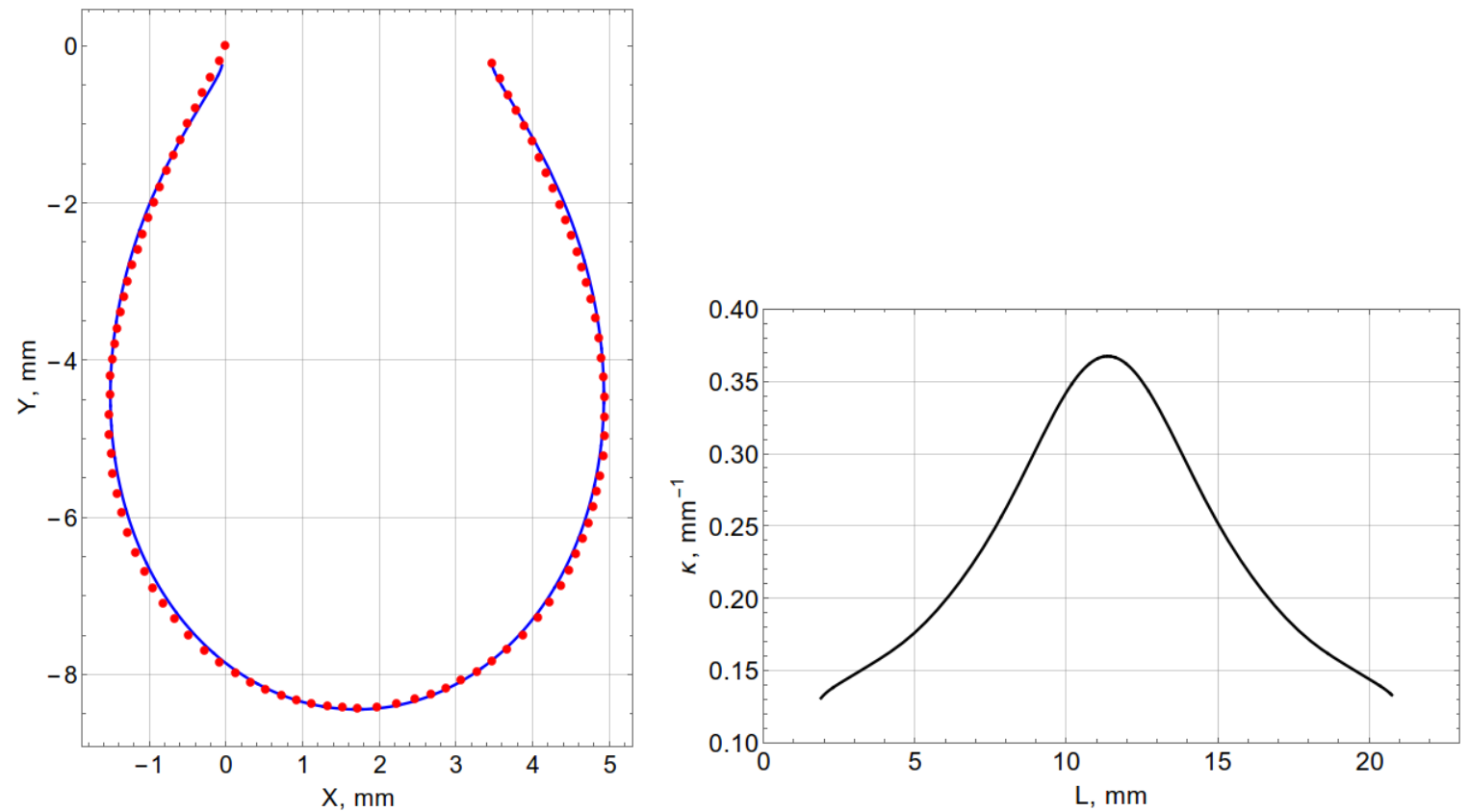

Figure 5. (a) Measured shape of curved fiber (red points) fitted with the curve determined by Eq. (4) - (blue curve). (b) The fiber curvature as a function of distance $\mathrm{L}$ along the fiber.

In Fig. 6 we rescaled the height of fiber curvature variation shown in Fig. 5(b) to fit the experimentally measured ERV. The comparison shown in Fig. 6 demonstrates that with a good accuracy the ERV of bent optical fiber linearly depends on the curvature of the loop.

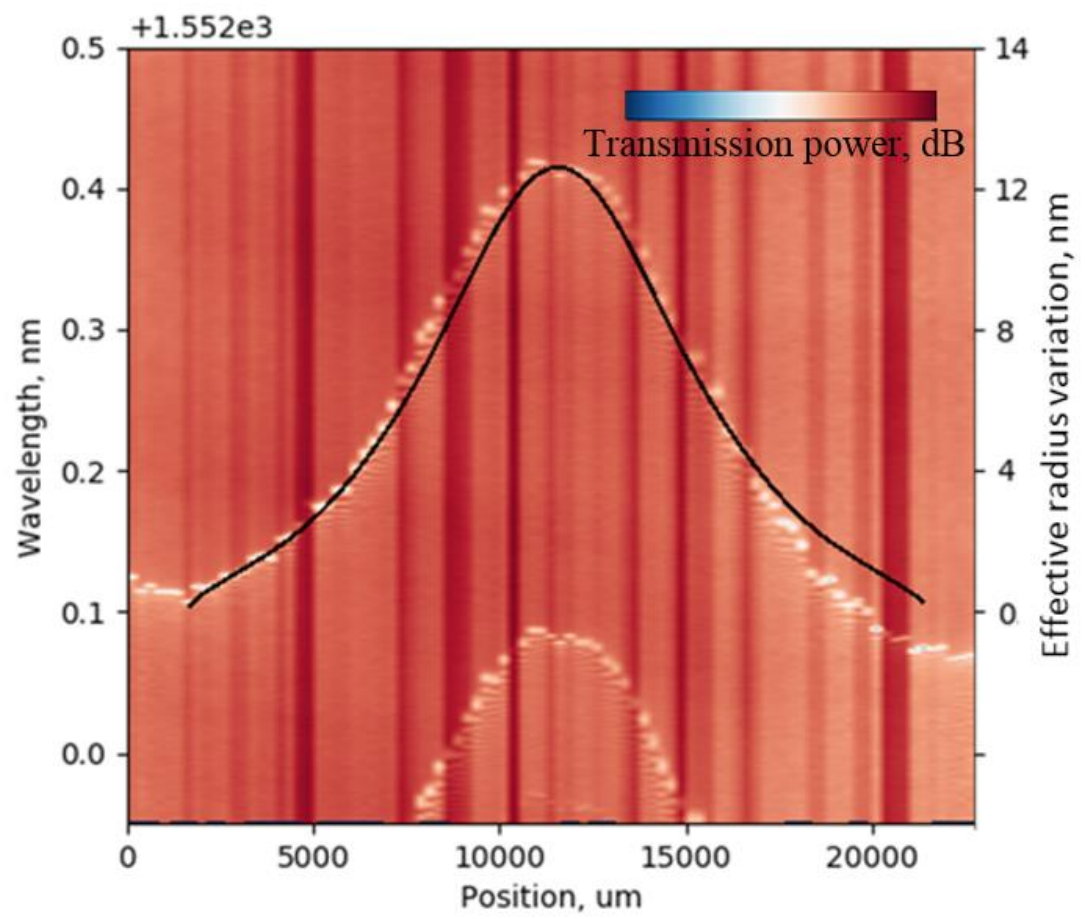

Fig. 6 Experimental characterization of the nanoscale effective radius variation and spectra of the fabricated SNAP structure introduced by bending of optical fiber. Black function represents the curvature of the fiber loop. 


\section{CONCLUSION}

We have demonstrated the possibility to fabricate SNAP microresonators by bending the optical fiber. In our first proof of concept experiment, a simplest optical bottle resonator with ERV of $\sim 10 \mathrm{~nm}$ was fabricated by bending a commercial $125 \mu \mathrm{m}$ diameter optical fiber. SNAP structures with more complex shapes can be fabricated by advanced bending techniques using specially prepared fiber bending setups. Our simulation results are in reasonable agreement with experimental observations. The simplicity of the developed method and the ability to tune the introduced resonant structures mechanically is of great importance for the creation of robust and tunable SNAP devices for applications in optical signal processing and ultraprecise sensing.

\section{ACKNOWLEDGEMENTS}

I.D.Vatnik acknowledges the support of Ministry of Education and Science of the Russian Federation (3.7672.2017/8.9).

M. Sumetsky acknowledges the Royal Society Wolfson Research Merit Award (WM130110) and support from the Engineering and Physical Sciences Research Council (EPSRC) (EP/P006183/1) and Horizon 2020 Framework Programme (H2020-EU.1.3.3, 691011).

N. A. Toropov acknowledges the support from the Horizon 2020 Research and Innovation Programme (MULTIPLY, 713694).

\section{REFERENCES}

[1] Sumetsky, M., "Nanophotonics of optical fibers," Nanophotonics 2, 393 (2013).

[2] Toropov, N. A., and Sumetsky, M., "Permanent matching of coupled optical bottle resonators with better than 0.16 GHz precision," Opt. Lett. 41, 2278 (2016).

[3] Shen, F., Shu, X., Zhang, L., and Sumetsky, M., "Fabrication of surface nanoscale axial photonics structures with a femtosecond laser," Opt. Lett. 41, 2795 (2016).

[4] Huang, M., "Stress effects on the performance of optical waveguides," International Journal of Solids and Structures 40(7), 1615-1632 (2003).

[5] Sokornikoff, I. S., "Mathematical theory of elasticity," McGraw-Hill, (1946).

[6] Sumetsky, M., DiGiovanni, D. J., Dulashko, Y., Fini, J. M., Liu, X., Monberg, E. M., and Taunay, T. F., "Surface nanoscale axial photonics: robust fabrication of high-quality-factor microresonators," Opt. Lett. 36, 4824 (2011).

[7] Knight, J. C., Cheung, G., Jacques, F., and Birks, T. A., "Phase-matched excitation of whispering-gallery-mode resonances by a fiber taper," Opt. Lett. 22(15), 1129-1131 (1997). 